\title{
Spontaneous coronary artery dissection: a rare cause of acute coronary syndrome
}

\author{
Parichart Junpaparp, Abel Romero-Corral, Seung Yoon Celine Lee, Christian Witzke
}

Department of Medicine, Albert Einstein Medical Center, Philadelphia, Pennsylvania, USA

Correspondence to Dr Parichart Junpaparp, junpapap@einstein.edu, toon_mu@hotmail.com

Accepted 18 February 2014

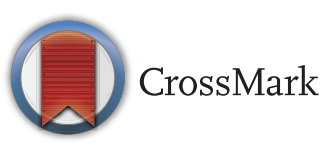

To cite: Junpaparp $P$, Romero-Corral A, Lee SYC, et al. BMJ Case Rep Published online: [please include Day Month Year] doi:10.1136/bcr-2013203243

\section{SUMMARY}

Spontaneous coronary artery dissection (SCAD) is a rare cause of acute myocardial infarction, which should always be included in differential diagnoses of acute coronary syndrome among young patients without known atherosclerotic risk. Although, it is commonly observed in young women during postpartum period, there were cases reported in connective tissue disorders, eosinophilic arteritis, contraception use, cocaine abuse and repetitive chest trauma. We report a case of SCAD, presenting with acute ST-elevation myocardial infarction, which was successfully treated conservatively.

\section{BACKGROUND}

Spontaneous coronary artery dissection (SCAD) is a rare cause of acute coronary syndrome or sudden cardiac death. It has been commonly described among young women during the early postpartum period. Other causes include repetitive chest trauma, strenuous exercise, cocaine abuse, eosinophilic coronary arteritis (Churg-Strauss syndrome, polyarteritis nodosa, Takayasu arteritis) and connective tissue disorder (Vascular Ehlers-Danlos syndrome, Marfan's syndrome). A spontaneous coronary dissection was once reported as a consequence of myocardial bridging in 2008 . Myocardial bridging has been considered as a benign congenital anomaly of the coronary artery which is characterised by an intramyocardial course of epicardial coronary artery. Although most patients remain asymptomatic, it can contribute to acute myocardial ischaemia, arrhythmia, atrioventricular block, syncope or sudden cardiac death in some cases. We report a case of SCAD at mid left anterior descending (LAD) artery with the presence of myocardial bridging at the distal segment. With a similar anatomical involvement, there might be a relationship between the myocardial bridging and SCAD. However, further observation is warranted to elucidate this hypothesis.

\section{CASE PRESENTATION}

A 25-year-old man with a medical history of wellcontrolled asthma presented to the emergency department with a sudden onset of chest tightness lasting for $30 \mathrm{~min}$ associated with light-headache, palpitation and diaphoresis. The patient denied any history of smoking, substance abuse or chest trauma. There was no familial history of sudden cardiac death, premature coronary artery disease or connective tissue disorders. Review of systems was negative for other organ system involvement. Physical examinations were unremarkable. Initial
ECG demonstrated ST-segment elevation at V1-2 (figure 1). The patient underwent emergency cardiac catheterisation which revealed a myocardial bridging of mid-LAD with a $25 \mathrm{~mm}$ length dissection located proximally (figure 2). An intravascular ultrasonography revealed luminal reduction with the presence of intimal flap and contrast-filled false lumen. Multiple attempts to place a stent were unsuccessful due to the difficulty of proper guide wire placement. As there was a patent flow observed throughout LAD (thrombolysis in myocardial infarction (TIMI)-3) and concern for false lumen cannulation, the patient was thus conservatively treated with aspirin and clopidogrel. At $24 \mathrm{~h}$, a follow-up CT angiography of coronary arteries did not reveal any flap propagation (figure 3). Further investigations were negative for serum antineutrophilic cytoplasmic antibody (ANCA) and COL3A1 gene mutation. The patient was chest pain free and subsequently discharged.

\section{DISCUSSION}

SCAD is a rare cause of acute coronary syndrome or sudden cardiac death. It has been commonly described among young women during the early postpartum period. The presumably hypertensive trauma during childbirth, increased shear stress on the arterial wall, decreased collagen production and haemorrhagic disruption of the vasa vasarum are the contributing factors to an intimal tear and cause blood entry into the media. ${ }^{1}$ Dissection is commonly observed at LAD (75\%) and right coronary artery $(20 \%)$. Other causes include repetitive chest trauma, strenuous exercise, cocaine abuse, eosinophilic coronary arteritis (Churg-Strauss syndrome, polyarteritis nodosa, Takayasu arteritis) ${ }^{2}$ and connective tissue disorder (Vascular Ehlers-Danlos syndrome, Marfan's syndrome). ${ }^{3} 4$ This patient developed spontaneous coronary dissection without any history of substance abuse, chest trauma or strenuous exercise. A physical examination did not reveal clinical features of connective tissue disorders or other organ system involvement. Genetic tests were also negative for gene mutations (COL3A1 gene) which excluded vascular Ehlers-Danlos syndrome. With an absence of eosinophilia, as well as, negative C-ANCA and P-ANCA made eosinophilic coronary vasculitis unlikely. Therefore, the cause of SCAD remained unclear. With the presence of myocardial bridging located distally to the dissecting segment, it was suspicious that there might be a relationship between the two conditions in this case. 


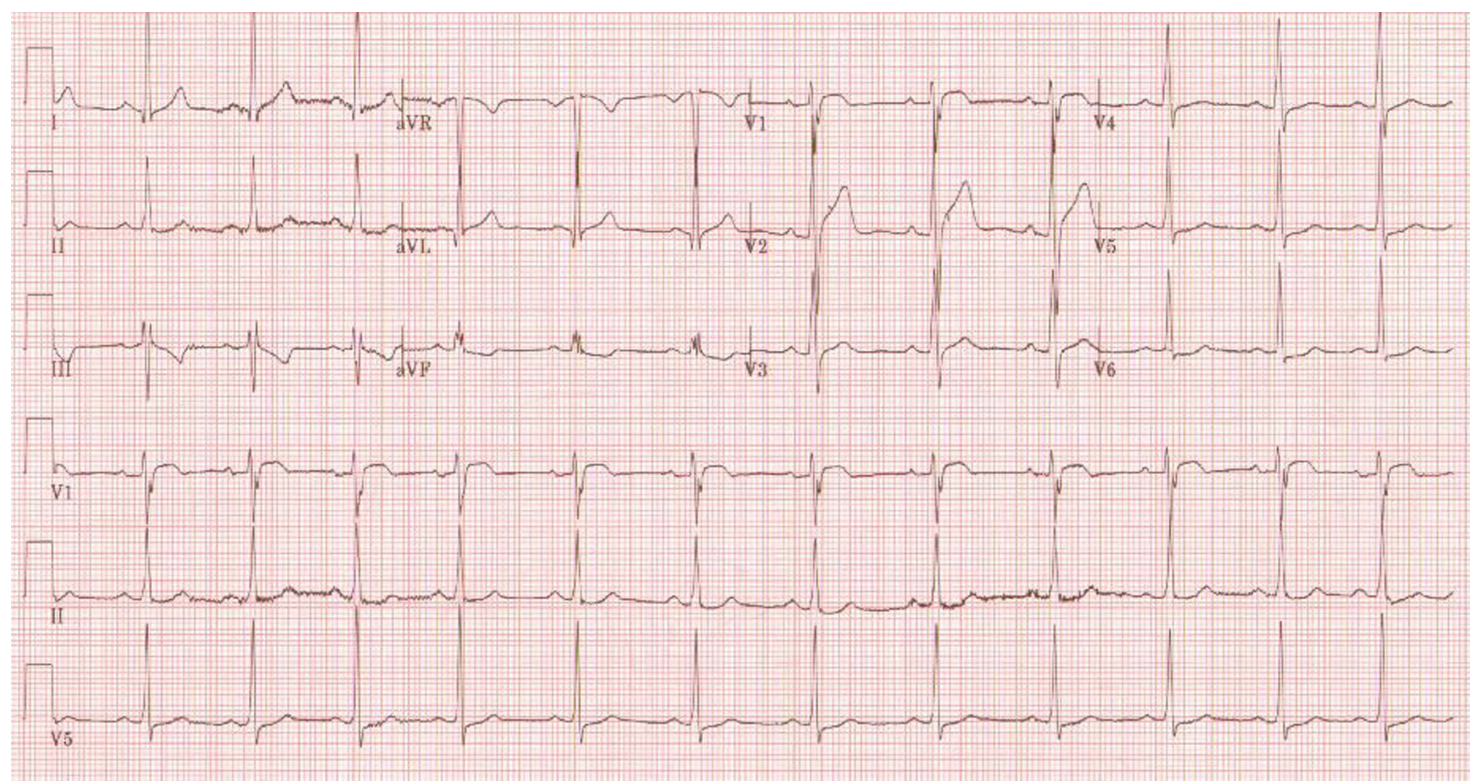

Figure 1 Admission ECG demonstrated ST-segment elevation of V1-V2.

A spontaneous coronary dissection was first reported as a consequence of myocardial bridging by Ge et al. ${ }^{5}$ Myocardial bridging is a congenital anomaly of the coronary artery which is characterised by intramyocardial course of epicardial coronary artery. The prevalence is less than $5 \%$ as reported by coronary angiography but much higher among autopsy reports. Myocardial bridge is commonly localised at mid-LAD. Typically, it has been considered to be a benign condition in which most patients remain asymptomatic. In some cases, it can contribute to acute myocardial ischaemia, arrhythmia, atrioventricular block, syncope or sudden cardiac death. ${ }^{6}$ The pathophysiology of ischaemia includes luminal narrowing during systole and formation of atherosclerosis in the segment proximal to the bridge. The concomitant of SCAD and myocardial bridge in the same coronary artery was only reported in one case. Though, the pathogenesis of SCAD is uncertain, it is likely related with the difference of shear force between proximal artery and the bridge tunnel. The typical coronary angiographic findings of myocardial bridging are 'Milking effect' and a 'step down-step up phenomenon' which represent the systolic narrowing of the tunnelled

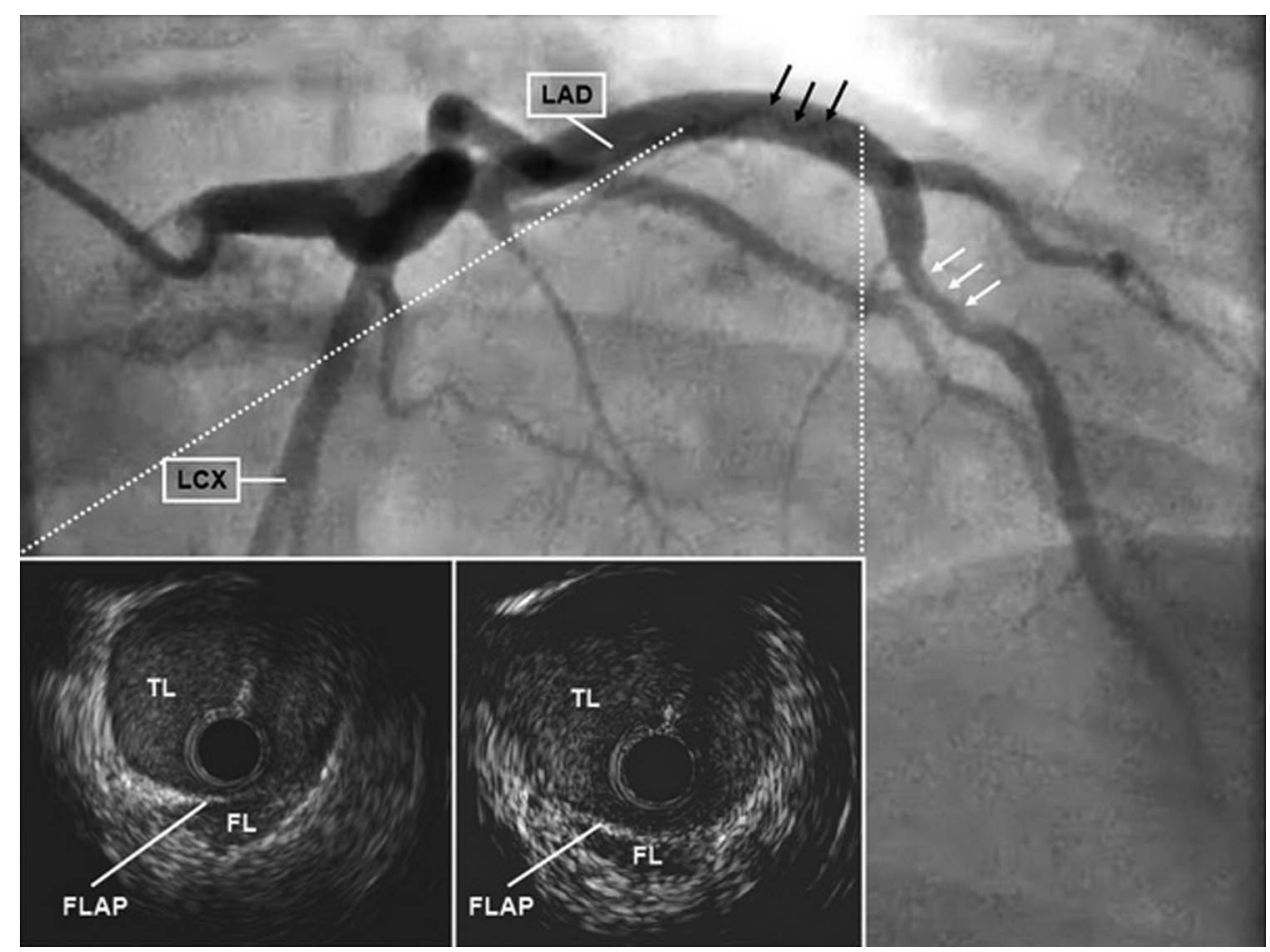

Figure 2 Coronary angiography revealed a myocardial bridging (white arrow) at mid left anterior descending with a $25 \mathrm{~mm}$ length dissection (black arrow) located proximally. The intravascular ultrasonography revealed luminal reduction of true lumen (TL) with the presence of intimal flap and contrast-filled false lumen (FL). 
Figure 3 CT angiography coronary artery revealed short, linear filling defect in the mid left anterior descending (LAD) immediately past of first septal perforator compatible with a small dissection flap. The flap does not propagate to the proximal LAD or left main artery.

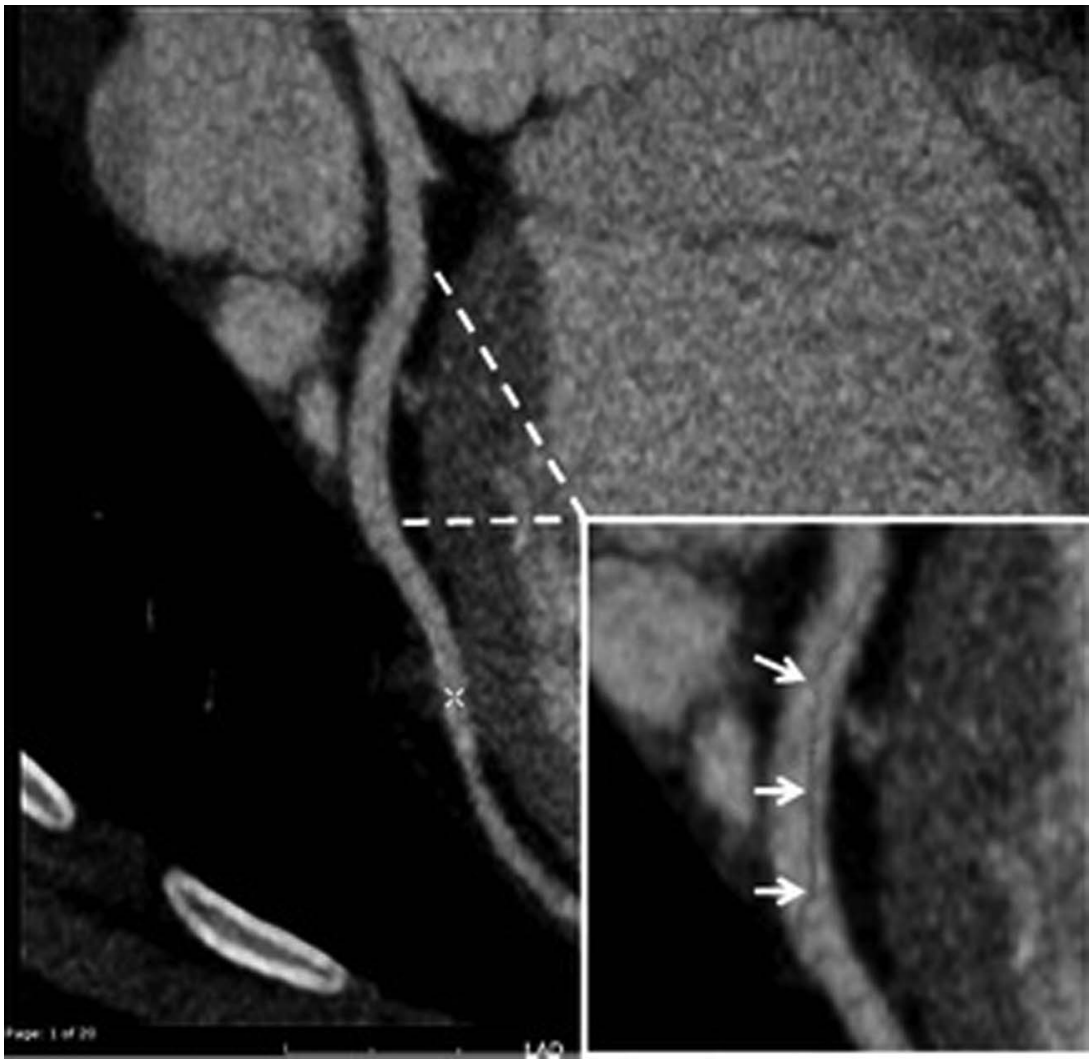

segment. With an application of intravascular Doppler ultrasound, the typical 'Half-moon phenomenon', which is an echolucent area surrounding the bridge, is being observed throughout the cardiac cycle. Moreover, a characteristic flow pattern in the bridge segment includes a very high flow velocity at early diastole followed by a sharp deceleration and subsequent plateau, so-called 'finger-tip' phenomenon. ${ }^{89}$

The treatment of SCAD includes conservative medical therapy, percutaneous coronary intervention (PCI) and coronary artery bypass surgery (CABG). A thrombolytic therapy should be avoided as it can increase extent of disease. ${ }^{10}$ To date, there is no definite treatment guideline in SCAD. The treatment of choice depends on clinical symptoms, extent and location of the dissection. According to SCAD case series by Tweet et al, conservative management was highly recommended in patients with preserved coronary blood flow. The CABG in selected cases, left main coronary or multivessels dissection, was also associated with satisfied clinical outcome. However, it could not provide protective effect against recurrent dissection. Given the difficulty crossing wire into true lumen and potential propagation of dissection during intervention, the technical success in PCI is only $65 \% .{ }^{11}$ In this patient, dissection was localised at mid-LAD with patent flow (TIMI-3), she was therefore treated conservatively with dual antiplatelet. With a concomitant myocardial bridging, an application of calcium channel blocker would provide additional benefit against angina chest pain. Importantly, nitrate should be avoided in this case as it can augment the systolic narrowing of the myocardial bridge by increasing vessel compliance. ${ }^{12}$ At long-term follow-up, the 10 -year recurrent rate of SCAD was $29 \%$, more commonly among female patients.

This case illustrated a rare cause of acute coronary syndrome in a young healthy individual and demonstrated the potential relationship between myocardial bridging and SCAD, which may raise more concerns on this benign coronary variant in the future.

\section{Learning points}

- Spontaneous coronary artery dissection (SCAD) is one of the rare causes of acute coronary syndrome, particularly among young healthy individuals.

- SCAD is commonly observed in the postpartum period, in patients with chest trauma, strenuous exercise, cocaine abuse, eosinophilic coronary arteritis and connective tissue disorders.

- Although, myocardial bridging has been considered as a benign variant, the true relationship with coronary dissection needs to be elucidated with further study.

- Treatment of SCAD includes conservative medical management, coronary angioplasty and coronary artery bypass surgery. The treatment selection should be made on the clinical symptoms, extent and location of dissection, as well as the preservation of distal coronary flow.

Contributors All authors were involved in manuscript preparation and patient care. Competing interests None.

Patient consent Obtained.

Provenance and peer review Not commissioned; externally peer reviewed.

\section{REFERENCES}

1 Biswas M, Sethi A, Voyce SJ. Spontaneous coronary artery dissection: case report and review of literature. Heart Views 2012;13:149-54

2 Lepper PM, Koenig W, Moller P, et al. A case of sudden cardiac death due to isolated eosinophilic coronary arteritis. Chest 2005;128:1047-50.

3 Hampole CV, Philip F, Shafii A, et al. Spontaneous coronary artery dissection in Ehlers-Danlos syndrome. Ann Thorac Surg 2011;92:1883-4.

4 Nakamura M, Yajima J, Oikawa Y, et al. Vascular Ehlers-Danlos syndrome-all three coronary artery spontaneous dissections. J Cardiol 2009;53:458-62. 
5 Ge JB, Huang ZY, Liu XB, et al. Spontaneous coronary dissection associated with myocardial bridge causing acute myocardial infarction. Chin Med J (Engl) 2008:121:2450-3.

6 Duygu H, Zoghi M, Nalbantgil S, et al. Myocardial bridge: a bridge to atherosclerosis. Anadolu Kardiyol Derg 2007:7:12-16.

7 Mohlenkamp S, Hort W, Ge J, et al. Update on myocardial bridging. Circulation 2002;106:2616-22.

8 Ge J, Jeremias A, Rupp A, et al. New signs characteristic of myocardial bridging demonstrated by intracoronary ultrasound and Doppler. Eur Heart J 1999;20:1707-16.
9 Ripa C, Melatini MC, Olivieri F, et al. Myocardial bridging: a 'forgotten' cause of acute coronary syndrome - a case report. Int J Angiol 2007;16:115-18.

10 Mahadevappa N, Singh B, Bhairappa S, et al. Spontaneous multivessel coronary artery dissection with anomalous coronary artery. BMJ Case Rep 2013;2013.

11 Tweet MS, Hayes SN, Pitta SR, et al. Clinical features, management, and prognosis of spontaneous coronary artery dissection. Circulation 2012;126:579-88.

12 Berry JF, von Mering GO, Schmalfuss C, et al. Systolic compression of the left anterior descending coronary artery: a case series, review of the literature, and therapeutic options including stenting. Catheter Cardiovasc Interv 2002;56:58-63.

Copyright 2014 BMJ Publishing Group. All rights reserved. For permission to reuse any of this content visit

http://group.bmj.com/group/rights-licensing/permissions.

BMJ Case Report Fellows may re-use this article for personal use and teaching without any further permission.

Become a Fellow of BMJ Case Reports today and you can:

- Submit as many cases as you like

- Enjoy fast sympathetic peer review and rapid publication of accepted articles

- Access all the published articles

- Re-use any of the published material for personal use and teaching without further permission

For information on Institutional Fellowships contact consortiasales@bmjgroup.com

Visit casereports.bmj.com for more articles like this and to become a Fellow 